\title{
BMG sieht keine Datenschutzverletzung bei den AKR
}

- Mit Schreiben vom 7. Dezember 2010 hat das Bundesgesundheitsministerium (BMG) einen Bericht zur datenschutzrechtlichen Unbedenklichkeit des aus den neuen ambulanten Kodierrichtlinien (AKR) resultierenden Datentransfers vorgelegt. Nach Auffassung des Ministeriums vereinbaren die Bundesmantelvertragspartner nach \$295 Abs. 3 SGB V eigenverantwortlich Richtlinien für die Vergabe und Dokumentation der Diagnosenschlüssel für die Abrechnung und Vergütung der vertragsärztlichen Leistungen (Ambulante Kodierrichtlinien, AKR). Diese $A K R$ wären dem BMG vorgelegt worden und die Vereinbarungen wäre dort nicht beanstandet worden.

Die AKR würden für eine korrekte und eindeutige Diagnosenverschlüsselung und damit eine Grundvoraussetzung für die an der Morbiditätsentwicklung ausgerichtete Vergütungsdynamisierung für die Vertragsärzte sowie den morbiditätsorientierten Risikostrukturausgleich (Morbi-RSA) stehen. Kodierregeln bestünden außerdem seit Jahren auch im stationären Bereich bei den Fallpauschalen (DRGs). Die Einschätzung, nach der die AKR einen Eingriff in den Datenschutz darstellen und dazu führen könnten, dass Patienten aus diesen erfassten Daten z.B. beim Abschluss von Lebensversicherungen ein Nachteil erwächst, teilt das BMG nicht.

Die AKR sind nach dessen Auffassung dem Vertragsarzt eine Hilfe, korrekt und eindeutig die im aktuellen Quartal gestellten Behandlungsdiagnosen zu verschlüsseln. Sie begrenzten dazu die Übermittlung der Diagnosen auf den für Abrechnungszwecke erforderlichen Umfang und würden dafür sorgen, dass z.B. eine Übermittlung von anamnestischen Diagnosen ohne Leistungsbezug im abzurechnenden Quartal sowie abnorme Befunde ohne weiterführende Diagnostik oder Therapie unterbleiben. Insoweit

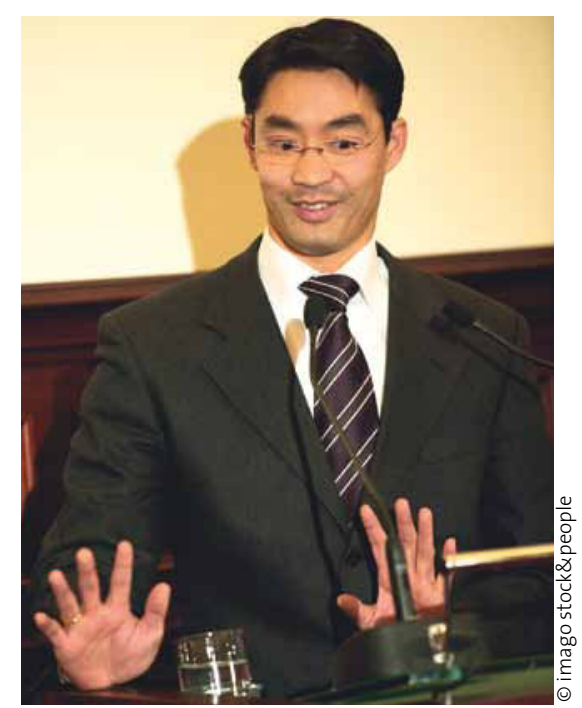

Gesundheitsminister Rösler: „Bürokratiemessung" angekündigt.

würde den Erfordernissen des Datenschutzes künftig besser Rechnung getragen. Im Übrigen handele es sich bei im Zusammenhang mit den AKR erhobenen Daten um solche, die Abrechnungszwecken der gesetzlichen Krankenversicherung dienen und nur an Kassenärztliche Vereinigungen und gesetzliche Krankenkassen übermittelt werden dürfen.

Diese Daten unterlägen somit den sozialdatenschutzrechtlichen Vorgaben des Fünften und Zehnten Sozialgesetzbuches. Krankenkassen und Kassenärztliche Vereinigungen wären daher nur im Rahmen dieser Vorgaben befugt, personenbezogene Daten der Versicherten zu den gesetzlich genannten Zwecken (z.B. zur Abrechnung von Leistungen) in dem hierzu erforderlichen Umfang zu nutzen. Eine Weitergabe an kooperierende private Organisationen oder den Arbeitgeber sei datenschutzrechtlich unzulässig. Eine Kenntnisnahme von Unternehmen, die Lebensversicherungen anbieten, oder Arbeitgebern im Rahmen eines Bewerbungsverfahrens sei damit ausgeschlossen.
Die Stellungnahme des BMG stützt sich auf Angaben der KBV, wonach den Vertragsärzten und Vertragspsychotherapeuten für die Zeit vom 1. Januar 2011 bis zum 30. Juni 2011 eine Übergangs- bzw. Einführungsfrist eingeräumt wurde, um sich mit den AKR vertraut zu machen, ohne dass Sanktionen drohen. Innerhalb dieser Frist könnten Ärzte und Psychotherapeuten selbst entscheiden, ab wann sie die AKR anwenden wollen. Grund für die Übergangsphase wären die Ergebnisse des in Bayern durchgeführten Praxistests, die Potentiale zur Verbesserung der Einführung der AKR in den Praxisalltag aufgezeigt hätten. Zusätzlich würde die KBV die Einführung der AKR durch eine Bürokratiemessung begleiten, um Belastungen zu identifizieren und zu minimieren sowie zur Bearbeitung von Fragen der Anwendung der AKR in Hausarztpraxen zusammen mit dem Zentralinstitut für die kassenärztliche Versorgung (ZI) eine Expertengruppe einsetzen.

\section{MMW Kommentar}

Die Stellungnahme des BMG beinhaltet gleich mehrere beachtenswerte Elemente. Zum ersten kommt diese Stellungnahme, bevor die mit der Frage der Datenschutzkonformität konfrontierten Datenschutzbeauftragten der Länder bzw. des Bundes Stellung bezogen haben. Auch muss nach dem Briefinhalt jeder Vertragsarzt künftig die Auskunft an z.B. private Lebens-, Kranken- oder Unfallversicherungen verweigern, denn eine solche Weitergabe ist nach Auffassung des BMG datenschutzrechtlich grundsätzlich nicht zulässig. Bemerkenswert ist schließlich noch der Hinweis, dass KBV und Zl eine „Bürokratiemessung "im Zusammenhang mit der Einführung der AKR durchführen. Über einen solchen Feldversuch ist wenige Tage vor Beginn der Einführung weder eine entsprechende Testgruppe noch ein Testversuchsdesign bekannt. 